\title{
7. FLASH STARS IN STELLAR AGGREGATES
}

\author{
Guillermo Haro \\ Tonantzintla and Tacubaya Observatories
}

In the past 10 years numerous extremely rapid variables have been found in different stellar associations. The spectrophotometric characteristics of some of these explosive variables are so similar to those of the UV Ceti stars that we propose the term "flash stars" for this variety, in order to provisionally distinguish them from the classical flare stars.

At present we know about 130 dwarf stars with outbursts of remarkably short duration that appear to be associated with stellar aggregates such as that of the Orion nebula, of the Taurus dark clouds, and of NGC 2264. The observational data relating to these variables enable us to formulate a more adequate criterion for the classification of flash stars in nebulae. Our aim in sketching here this new criterion is to improve upon our former definition (Haro 1957) and avoid - as far as possible - confusion with other types of variables which, in certain aspects, resemble the flash stars.

\section{Photometric Properties of Flash Stars}

(1) They exhibit very short-lived and non-periodic outbursts lasting from a few minutes to approximately $3 \mathrm{hr}$. There are few cases in which the total time of variation is as long as $6 \mathrm{hr}$.

(2) The light curves are singular; the star remains most of the time near its normal minimum brightness, but suddenly and unpredictably, in a matter of seconds or very few minutes, it flares up; the decline from maximum may be slower, but it is also rapid. In some cases, a first outburst is followed immediately by a second one.

(3) The amplitude of the outburst is always much greater in the ultraviolet than it is in blue, yellow, or red light.

(4) In the near infrared ( $8400 \AA)$ the flash variation is beyond the range of sensitivity of our photographic method, even in those cases where the amplitude in the ultraviolet is of the order of 4 to 6 magnitudes.

(5) The total duration of a flash - from normal minimum to maximum and back to minimum - does not seem to be related, in all cases, to the amplitude of the variation, although in some stars the larger the amplitude the greater the total time of variation.

\section{Spectral Properties of Flash Stars} late $\mathrm{dM}$.

(1) The spectral types of the flash stars range from at least dK2 down to

(2) The majority of the flash stars do not show - on objective prism plates and during minima - emission lines, although in about $30 \%$ of the cases the emission lines are weak or only moderately strong. 
(3) The appearance or the enhancement of emission lines is observed during maximum light and especially right after maximum is reached.

(4) During an outburst, as far as can be inferred from the rather limited observational material, spectral characteristics cannot be distinguished from those of some T-Tauri stars; in addition to the bright line spectrum, a strong continuous emission is present, especially in the ultraviolet.

(5) Flash stars that at their normal minima do not show emission lines may retain weak emission lines for the first day or two of the minimum following outburst.

Among the stars that, according to the criteria just given, qualify as flash stars there is still a wide variation as regards individual spectrophotometric features. The extremes of this variation may be characterized as follows under $(a)$ and $(b)$.

(a) During the outburst only the ultraviolet and part of the blue continuum are enhanced. The emission lines are extremely strong, but the red continuum does not seem to be affected. There is no variation in the near infrared.

(b) During the brightening not only the violet and blue continuum increase, but also the apparent red continuum. Although the variation in the ultraviolet is by far the greater, the red continuum is conspicuously enhanced. In contrast, the emission lines are only weak or moderately strong. As under $(a)$ there does not seem to be any variability in the near infrared.

The striking difference between cases $(a)$ and $(b)$ is illustrated in Figure 1. Flash star No. 8 in Orion (Haro and Rivera 1954) also illustrates case (b).

One can speculate about these two extreme spectrophotometric features of the flash stars following Ambartsumian's ideas (1954, 1957), to the effect that if the flash phenomenon takes place at different layers of the outer envelope of the star there would be marked spectroscopic differences, which could depend on the depth of the explosion. If such is the case, it would be expected that when the explosion takes place near the photospheric layers one should observe a mixture of thermal and nonthermal radiation. Consequently, the infrared continuum must also be enhanced. But as we have seen, no infrared intensification is noticed during the flash.

An alternative would be to assume that the flash explosion always takes place in the stellar chromosphere and that the spectroscopic differences among the flash stars are mainly due to different degrees of excitation. Then, the apparent intensification of the red continuum on our small dispersion spectra might be caused either by the extension of the nonthermal continuum or by the enhancement of complex blends of emission lines, possibly of metals. But also here we encounter the difficulty of explaining why in flash stars showing intensification in the red part of the spectrum the normal emission lines are so inconspicuous. The only way to settle the question would be through the study of high dispersion spectrograms of these stars during the flash.

We believe that the aforementioned spectrophotometric characteristics will suffice for the unambiguous assignment of a variable to the flash type. Even in such a difficult and general case as that of the T-Tauri and RW Aurigae stars, our criteria enable us to distinguish the latter from the flash stars: firstly, because the flash objects show a unique shape of light curve and, secondly, because they show character- 
istic differences of amplitude variation in the near infrared. As regards the T-Tauri variables, the irregular and relatively smooth variation can easily be detected in the near infrared but, apparently, this is not true of the flash stars. In this connection one should bear in mind that in some stars the flash variation may appear superposed on the irregular "normal" T-Tauri type variation; even in these cases, the "flash" peculiarity can be discriminated by the form of the light curve combined with the infrared observations.

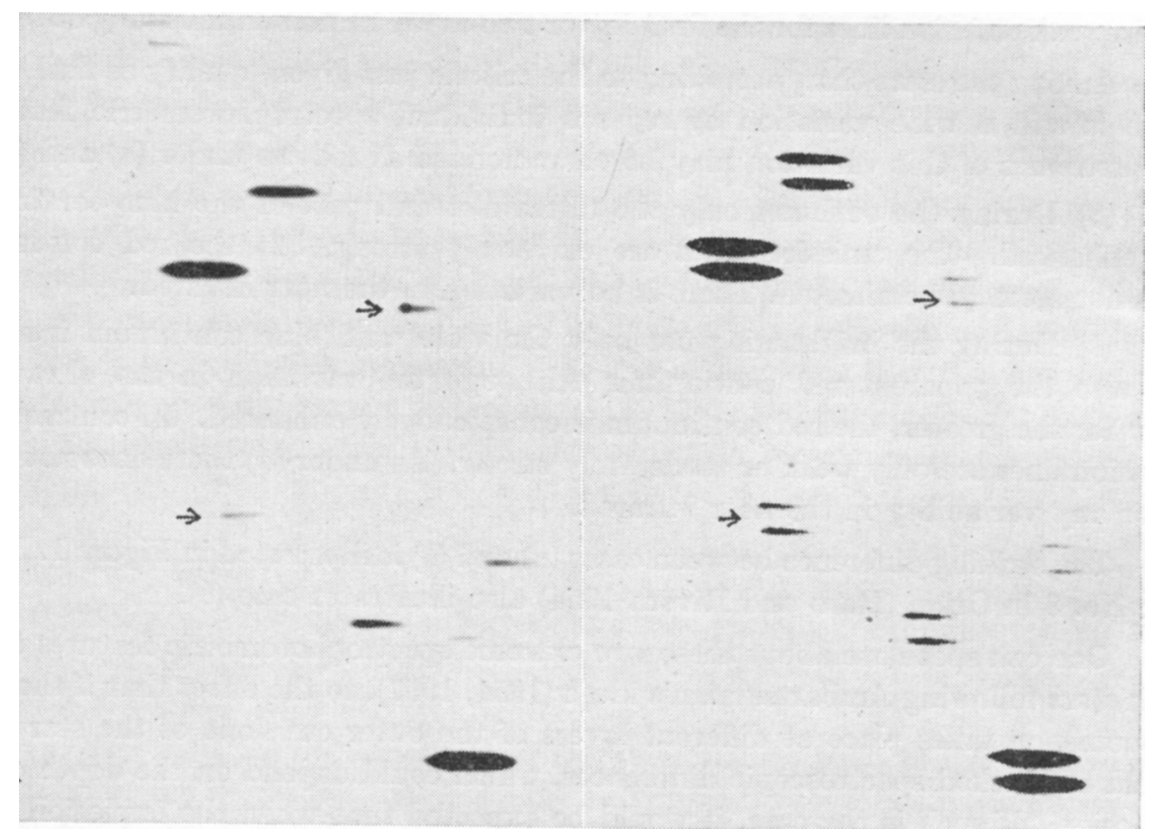

Fig. 1.-On these two spectral photographs (spectral range from $6100 \AA$ to $\mathbf{H} \alpha$ ) of which the right one has double images, the two extreme examples of the spectroscopic behaviour of flash stars are illustrated. The upper marked object shows an extremely strong $\mathrm{H} \alpha$ in emission during the flare and no continuum enhanced; the lower one shows conspicuous intensification of the apparent continuum but very weak emission $\mathrm{H} \alpha$ line. For identification of the field the three brightest stars are Parenago Nos. 2342, 2420, and 2433.

In fact, at the Tonantzintla Observatory we undertook the survey of flash stars in associations, inspired by Joy's (1949) and by our own observations (Haro 1953) of some T-Tauri stars that show extremely abrupt and conspicuous outbursts. Thus, we discovered the first "pure" flash type stars in the Orion nebula; by "pure" we mean stars that show the flash phenomenon, but cannot be normally classified as T-Tauri stars because they lack during minima the appropriate spectroscopic features.

We were thus led to contemplate the possibility that the flash stars may be members of the wider T-Tauri family; they may represent-we speculate by-products or a later stage of the T-Tauri stars in their evolution toward the main sequence. So we predicted as a plausible conclusion that wherever there exists a group of T-Tauri like objects, that is a T-association, it should be possible to find 
rapid variables of the flash type in such associations. I personally believe that this prediction has been fully confirmed, mainly through our surveys and Rosino's observations.

\section{An Observational Approach to an Evolutionary Sequence $-A$ Working Hypothesis}

The data obtained on flash stars discovered in different associations make quite attractive the idea that the flash phenomenon, which probably is caused by some kind of nonthermal explosive process in the upper stellar chromosphere, can occur throughout the different stages of the evolution of a late dwarf star. Thus, we can arrange the observational results of our variables into the following sequence, which seems to have some evolutionary sense:

(1) Typical T-Tauri stars, in which the flash variation is occasionally superposed. Examples: DF Tau, DK Tau, YZ Ori, BW Ori.

(2) Dwarf late-type stars, in which the T-Tauri spectroscopic features are still present but considerably diminished and the outbursts due to sporadic flashes are the most conspicuous variations. Examples: V389 Ori, V390 Ori.

(3) The "pure" flash stars, in which the T-Tauri properties have practically disappeared, at least during the more or less permanent minimum; they resemble normal non-emission late-type dwarfs, except during and immediately following the flare. Examples: EY Tau, FF Tau, FH Tau, V386 Ori, V498 Ori, and the majority of flash stars in associations.

Now, let us adopt the view that in a stellar aggregate the formation of the stars is not coeval - an idea which recently has been brilliantly reinforced by Herbig (1962). We would then expect that in a given association not only the stars of different masses could have been formed at different epochs and therefore have different ages, but also - and especially in the case of stars of small mass - that these may have been formed in successive generations. It is thus conceivable that their spectrophotometric characteristics may reveal not only their age or generation, but also some relevant evolutionary features of the whole stellar aggregate to which they belong. For example, let us assume that (1) not all T-Tauri stars in an association were formed at the same time, and (2) that the "pure" flash stars represent the older and the more advanced evolutionary stage of a $\mathrm{T}$ Tauri. We would then have to conclude that in associations such as those of the Orion nebula, of the Taurus dark clouds, and in NGC 2264, there exist dwarf stars undergoing an explosive process of very different ages and evolutionary stages.

This general view has been presented in several of our previous papers (Haro 1957, 1962) as an attractive and plausible working hypothesis. The more we know about T-Tauri stars and flash variables, the more cogent are the arguments in favour of this point of view.

As a possible test of this working hypothesis, we can imagine a stellar aggregate old enough and at such an evolutionary stage that the typical T-Tauri stage has already been passed through and that the succeeding stage is represented by "pure" flash stars. A good example of this kind of aggregate would be the Pleiades, though undoubtedly it is not the only one. 


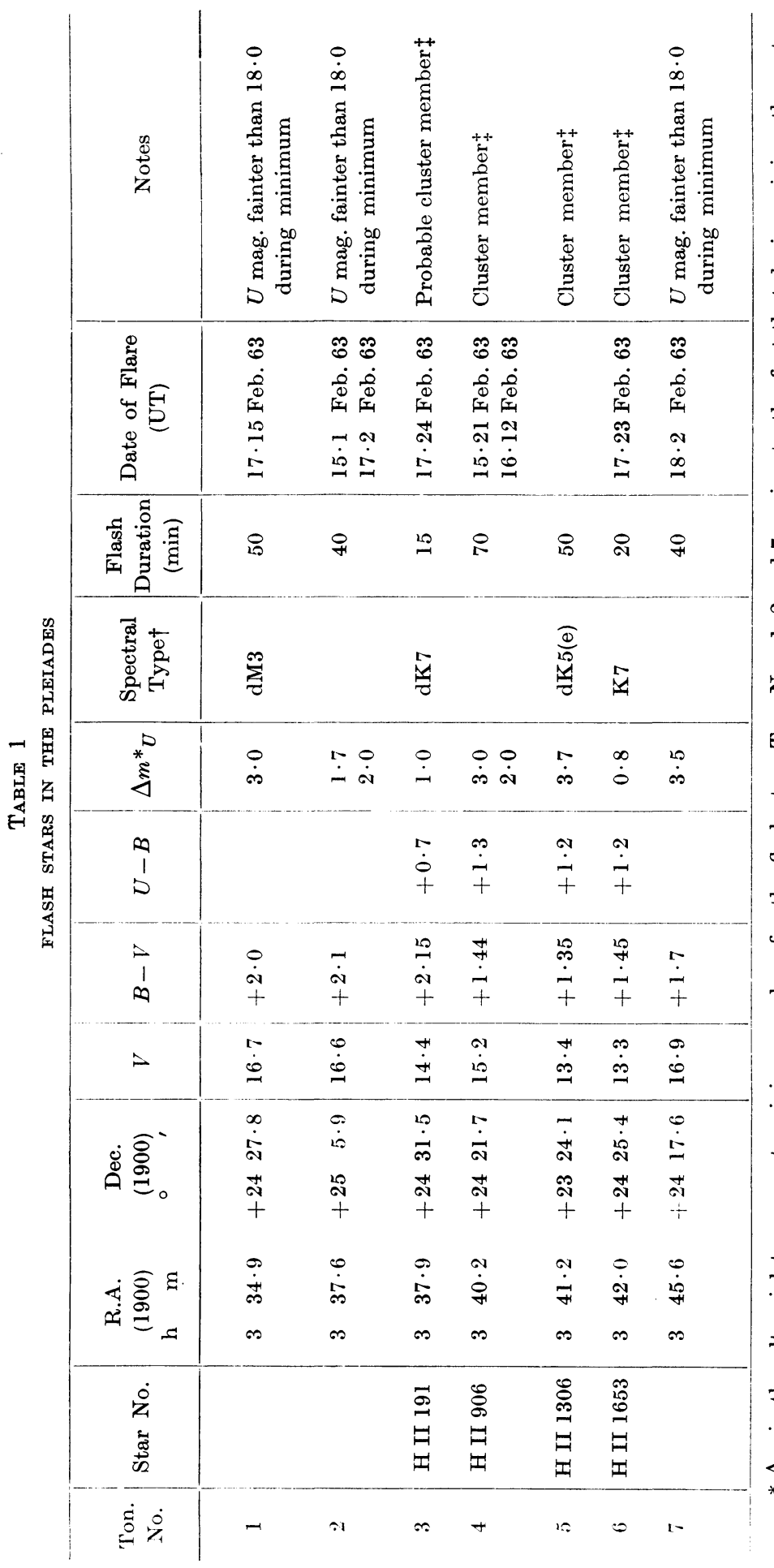

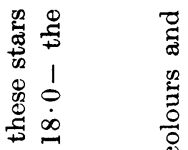

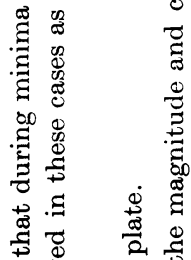

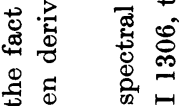

일

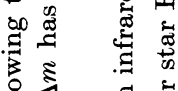

\&

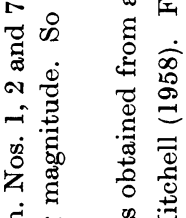

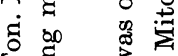

क .

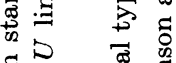

表市

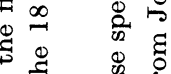

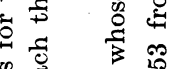

$\stackrel{\mathbb{D}}{\mathbb{0}} \cong$

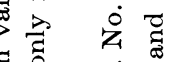

ह્વ

ส

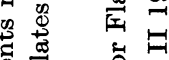

बै क्ष

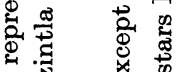

产

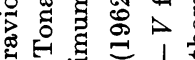
t5

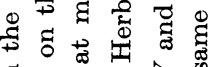

$\exists$ ⿷匚

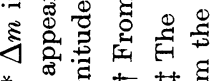


According to Herbig (1962), if the H-burning age of $6 \times 10^{7}$ years is applicable to the Pleiades, we would find stars fainter than about G2 still above the main sequence; yet he says that no systematic elevation of the main sequence is observed at least as far as type K5. At this point the classical Kelvin time would be of the order of $2 \times 10^{8}$ years. Furthermore, Herbig's observations establish quite clearly that there is no trace of T-Tauri stars or dMe stars among the faint members of the Pleiades to about the 16th visual magnitude. The writer has searched for dMe stars up to the 17th visual magnitude with the same negative results. Nevertheless, as will be presently shown, numerous "pure" flash stars of spectral type dK5 and later still appear.

It is well known that Johnson and Mitchell (1958) quite accidentally found a flash star which is a member of the Pleiades cluster and they suspected flash variability in some other cluster members (H II 2082, 1332, 1553, and 2407). These discoveries led us to search further for flash stars in the Pleiades. The preliminary results are summarized in Table 1, and illustrated in Figure 2.

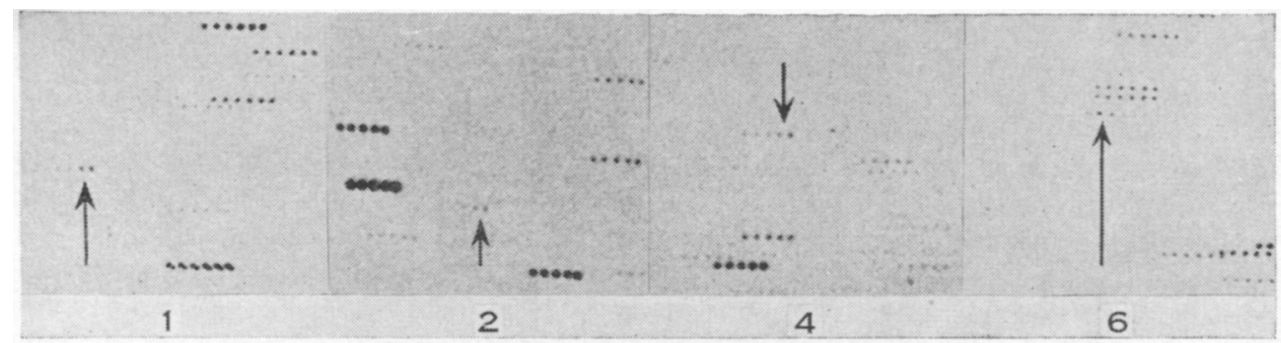

Fig. 2.-Examples of flash stars in the Pleiades during outbursts. From left to right the flash stars Ton. Nos. 1, 2, 4, and 6 of Table 1 are marked. Each exposure $=10^{\mathrm{m}}$, and the time separation between them $=1^{8}$.

In Table 1, the flash star of Johnson and Mitchell is also listed. We may mention that during our total time of effective observation $\left(24^{\mathrm{h}} 50^{\mathrm{m}}\right)$ we only detected a slight amplitude variation in H II 1306 but not in the other stars, in which these authors noticed magnitude and colour discrepancies in their repeated observations. On the other hand, we found that the new flash stars Ton. Nos. 2 and 4 developed two different and conspicuous outbursts during the first $9^{\mathrm{h}} 50^{\mathrm{m}}$ of patrol. That does not mean, of course, that one would have any doubts about the Johnson and Mitchell observations. The only obvious conclusion is that - based on Johnson and Mitchell's paper and on our preliminary results - the total number of flash stars in the Pleiades cluster must be quite large.

Up to what limit would we expect to find variable stars of the flash type in a stellar aggregate? Most probably up to the point where the explosive phase of the evolution of dwarf stars toward the main sequence has been completed. This statement could be tested in some of the older galactic clusters of the kind of the Hyades, Praesepe, NGC 752, M67, or, if necessary and possible, in some globular clusters.

Regarding the UV Ceti type stars found in the neighbourhood of the Sun, we do not see - as previously stated (Haro 1957, 1962) - any fundamental argument apart from their location, in favour of classifying them separately from the 
flash stars defined in this paper. In Table 2, we summarize some available data on the flash and UV Ceti type stars found in different stellar aggregates. I do believe that in itself this table is quite suggestive.

I wish to express my gratitude to Dr. Rupert Wildt for his valuable comments and suggestions, and also to Mr. Enrique Chavira who kindly took for me the Pleiades plates.

TABLE 2

FLASH STARS IN DIFFERENT STELLAR AGGREGATES

\begin{tabular}{|c|c|c|c|c|c|}
\hline Aggregate & $\begin{array}{c}\text { No. of } \\
\text { Flash } \\
\text { Stars }\end{array}$ & Spectral Types & $\begin{array}{c}\text { Total Duration } \\
\text { of the } \\
\text { Brightening } \\
\text { (min) }\end{array}$ & Maximum $\Delta m$ & Reference \\
\hline Orion nebula & 106 & $\mathrm{dK} 2$ to $\mathrm{dM}$ & $30-360$ & 6 & $1,2,3,4$ \\
\hline NGC 2264 & 12 & $\mathrm{k}-$ & $25-70$ & 3 & $5,6,7$ \\
\hline Taurus dark clouds & 11 & $\mathrm{dK} 6$ to $\mathrm{dM} 5$ & $10-60$ & 2 & 7,8 \\
\hline Pleiades & 7 & dK5 to dM3 & $15-70$ & $3 \cdot 7$ & $4,9,10$ \\
\hline $\begin{array}{l}\text { Solar neighbourhood } \\
\text { (UV Ceti type) }\end{array}$ & 20 & $\mathrm{dM} 0$ to $\mathrm{dM} 6$ & $3-300$ & 6 & $11,12,13$ \\
\hline
\end{tabular}

The references are as follows:

1. Haro et al. (1953, 1954a, 1960, 1962, 1963).

8. Haro and Chavira (1955).

2. Rosino et al. (1956, 1962).

9. Johnson and Mitchell (1958).

3. Dall'Olmo (1958).

10. This paper.

4. Herbig (1962).

11. Joy (1960).

5. Haro (1954b).

12. Oskanjan (1957a).

6. Rosino, Grubissich, and Maffei (1957).

13. Oskanjan (1957b.)

7. Petit (1958).

\section{References}

Ambartsumian, V. A. (1954).-Soob. Byurakan. Obs. No. 13.

Ambartsumian, V. A. (1957).—“Non-stable Stars." (Ed. G. H. Herbig.) Symp. IAU 3: $177-85$. [Dublin 1955.] (Cambridge Univ. Press.)

DaLl'almo, U. (1958).-Coelum 25: No. 1-2.

Haro, G. (1953).-Ap. J. $117: 73-82$.

Haro, G. (1954a).-Ton. y Tac., Bol. No. 11 : 11-18.

Haro, G. (1954b).-Personal communication to Rosino and Herbig.

Haro, G. (1957).— "Non-stable Stars." (Ed. G. H. Herbig.) Symp. IAU 3: 26-30. [Dublin 1955.] (Cambridge Univ. Press.)

Haro, G. (1962)._-“Symposium on Stellar Evolution." pp. 37-44. [La Plata Observatory 1960.] (Nat. Univ. La Plata Astron. Obs.)

Haro, G., and Chavira, E. (1955).-Ton. y Tac., Bol. No. 12 : 3-16.

Haro, G., and Chavira, E. (1963).-Still unpublished.

Haro, G., Chavira, E., and Mendoza, E. (1960).-A.J. 65: 490.

Haro, G., and Morgan, W. W. (1953).-Ap. J. 118: 16-17. 
Haro, G., and Rivera Terrazas, L. (1954).-Ton. y Tac., Bol. No. 10: 3-15.

Herbia, G. H. (1962).-Ap. J. 135 : 736-47.

Johnson, H. L., and Mrtchels, R. I. (1958).-Ap. J. 128 : 31-40.

Joy, A. H. (1949).-Ap. J. 110 : 424-37.

JoY, A. H. (1960)._-"Stars and Stellar Systems." (General Ed. G. P. Kuiper.) Vol. VI. pp. 653-75. (Univ. Chicago Press.)

Oskanjan, V. (1957a).—“Non-stable Stars." pp. 17-27. [Byurakan 1956.] (Acad. Sci. Armenian SSR: Erevan.)

OsKanjaN, V. (1957b). -Personal communication.

Petit, M. (1958)._-“Colloqui d'Astrofisica di Asiago (1956-1958).” Asiago Contr. No. 95: $29-44$.

Rosino, L. (1956).-Asiago Contr. No. 69.

Rosino, L., and Cian, A. (1962).-Asiago Contr. No. 125.

Rosino, L., Grubissich, C., and Maffei, P. (1957).-Asiago Contr. No. 82.

\title{
Discussion
}

Buscombe: Has any search been made for flash stars in II Scorpii, where Struve had previously found T-Tauri stars? Pleiades.

Haro: No, only in the Orion nebula, in NGC 2264, the Taurus dark clouds, and in the

Haro: I would like to state that the Tonantzintla Observatory and Sagamore Hill Radio Observatory have collaborated in observing flash stars in the Orion nebula. The purpose of the radio and optical observations over the Orion and Pleiades clusters, which will be continued at the end of the year, is to demonstrate that a radio outburst in a flash star can be detected as far as the distance of the Pleiades and the Orion nebula.

Slee: Simultaneous radio and optical observations made on the flare star V371 Orionis in November 1962 resulted in the detection of radio bursts at $75 \mathrm{~cm}$ and 15 metres. The results are at present provisional because the detailed light curve of the event, obtained with the BakerNunn Schmidt camera operated by the Smithsonian Institution at Woomera, is not yet available.

\section{GALACTIC EVOLUTION AND THE TWO-COLOUR DIAGRAM}

\author{
R. H. Stoy \\ Royal Observatory, Cape Town
}

Information about the past history of the Galaxy may be obtained from a two-colour diagram, since the present distribution of stars in such a diagram depends on the past rate of star formation and the past metal abundances in the interstellar medium. As an illustration of this, I would like to discuss three diagrams that were recently prepared by Dr. M. E. Dixon from Cape photometric data (Dixon $1963 a, b)$.

In the first diagram $(U-B)_{\mathrm{C}}$ is plotted against $(B-V)$ for all single stars of known MK type belonging to luminosity classes V and VI and lying within 100 pc of the Sun. Class VI is taken as referring to subdwarfs, particularly those with sufficiently extreme metal deficiencies to be detected as such by their spectra. The particular feature in this diagram to which $I$ wish to draw attention is the way in which the left-hand boundary of the domain in which the Class VI stars fall coincides with the "blanketing line" defined by

$$
(B-V)_{\text {Hyades }}=0 \cdot 60 \text {. }
$$

